\title{
NIÑOS QUE NARRAN: AUMENTO EN LA COMPLEJIDAD DISCURSIVA Y SINTÁCTICA DURANTE LA EDAD ESCOLAR?*
}

\author{
Maili Ow González ** \\ Carola Alvarado Barra***
}

\section{Resumen}

Este trabajo describe la producción narrativa oral a lo largo de la escolaridad, considerando complejidad sintáctica y rol de los paquetes clausulares (Katzenberger, 2004) en la secuencia narrativa (Adam, 1999). Se presentan resultados de una muestra de 259 escolares de distintos niveles y de una submuestra que considera el rol funcional de los paquetes y sus relaciones. Se confirman hallazgos previos: aumenta la complejidad sintáctica según edad y nivel escolar; varía la cantidad y uso funcional de paquetes clausulares y el manejo de la secuencia narrativa.

Palabras clave: desarrollo oral, complejidad sintáctica, narración, paquetes clausulares, secuencia narrativa.

\section{CHILDREN NARRATORS: DISCURSIVE AND SYNTACTIC COMPLEXITY GROWTH IN SCHOOL AGE}

\begin{abstract}
This paper describes oral narratives across schooling, considering syntactic complexity and the role of clause package (Katzenberger, 2004) in the narrative sequence (Adam, 1999). Results obtained from a sample of 259 Chilean students in various school grades are shown to describe syntactic complexity and a subsample of 15 texts was analyzed to detect the role of clause packagings and interclause relations. Results confirm previous findings: syntactic complexity increases as age and grade, increase the number and functional use of clause package and the mastery of the narrative sequence.
\end{abstract}

Keywords: oral development, syntactic complexity, narrative, clause packagings, narrative sequence.

Recibido: 16-01-2013 Aceptado: 16-04-2013

* $\quad$ Artículo de una investigación financiada por Proyecto FONDECYT 1100600. El desarrollo sintáctico tardío en la oralidad y las modalidades discursivas: hacia una visión funcional del desarrollo lingüístico en la edad escolar.

** Chilena, Doctora en Didáctica de la Lengua y Literatura, Pontificia Universidad Católica de Chile, Santiago, Chile.mow@uc.cl

*** Chilena, Magíster en Lingüística Aplicada, Pontificia Universidad Católica de Valparaíso, Valparaíso, Chile. carola_alvarado@mi.cl 


\section{Introducción}

Bruner (2004) considera dos maneras en las cuales el ser humano puede comprender y a su vez referir la realidad que lo circunda: un modo narrativo y un modo argumentativo. Desde el punto de vista ontogenético, el discurso narrativo es el primero que emerge antes de ingresar a la escolaridad (Bruner, 2004; Nippold, 1998). A pesar de que ha sido estudiado por diversos investigadores (Nelson, 1996; Ninio \& Snow, 1996) en cuanto discurso co-construido y andamiado por un adulto, para el español existen pocos estudios en esta línea (Sebastián \& Slobin, 1994; Uccelli \& Paez, 2007) entre los que cabe destacar los de Bocaz (1996) y Pavez, Coloma y Maggiolo (2009). Las investigaciones sobre producción autónoma de narraciones en edad escolar muestran que, desde $2^{\circ}$ grado, los niños tienden a organizar sus narrativas con un comienzo formal ["había una vez..."] y un cierre convencional ["y vivieron felices para siempre..."] (Jiménez, 2006).

Muchas de estas investigaciones han permitido entender aspectos centrales del desarrollo de lo narrativo en niños y adolescentes. Sin embargo, no permiten dar cuenta de la forma cómo se relaciona este incremento en el dominio del género con otros niveles del lenguaje, como lo es por ejemplo, la sintaxis. Es por ello que, para complementar esta visión, es necesario comprender cómo se complejiza la sintaxis en el discurso oral autónomo y cómo esta evolución se relaciona con las particularidades del discurso narrativo. Aun cuando se han llevado a cabo valiosos estudios sobre el desarrollo sintáctico tardío en narraciones orales que retoman las distinciones y aportes de Hunt (1965), la mayoría de estos han estado centrados en una aproximación autónoma, separada de la tipología textual, que cuantifica estructuras sintácticas complejas en función de la edad, tanto en relación con la presencia o ausencia de estos elementos, como su extensión y nivel de incrustación en el discurso.

Frente a lo planteado anteriormente, el objetivo de este trabajo es describir, desde una aproximación funcional discursiva (Berman, 2004; Nir-Sagiv \& Berman, 2009), la producción narrativa oral a lo largo de la escolaridad, considerando la complejidad sintáctica y el rol de los paquetes clausulares (Katzenberger, 2004) correspondientes a cada una de las fases de la secuencia narrativa (Adam, 1999). Para ello, las unidades básicas de análisis que se consideran en este trabajo son la cláusula y los paquetes clausulares que establecen diversos tipos de

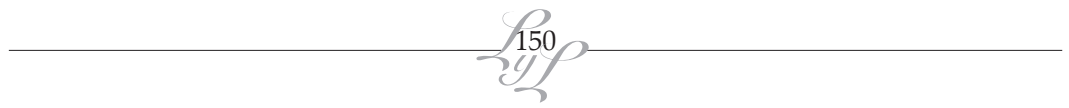


relaciones internas. Más que constatar la presencia de ciertas estructuras en el discurso narrativo oral de escolares de distintos niveles, se analiza el modo en que estas formas lingüísticas se relacionan y las funciones que cumplen. Para dar cuenta acerca de la complejidad sintáctica, se presentan los resultados de una muestra de 259 estudiantes de kínder, $3^{\circ}$ básico, $6^{\circ}$ básico, I medio y IV medio, de establecimientos educativos de dependencias públicas y privadas de la ciudad de Valparaíso, Chile. Por otra parte, se ha considerado una submuestra de 15 textos para dar cuenta del rol de los paquetes clausulares en la narración a partir de la secuencia narrativa de Adam (1999).

\section{Marco teórico}

\subsection{Estudios sobre desarrollo narrativo}

Dentro de los géneros discursivos, el discurso narrativo es el primero que emerge en los niños antes de ingresar a la escolaridad. Su estudio ha sido abarcado por diversos investigadores (Nelson, 1996; Ninio \& Snow, 1996). Sin embargo, se ha constatado que para el español existen pocos estudios en esta área. Cabe destacar los trabajos de Sebastián y Slobin, 1994; Bocaz, 1996; Uccelli y Páez, 2007; Pavez, Coloma y Maggiolo, 2009. El foco de análisis de un número importante de estas investigaciones ha estado puesto en las marcas de temporalidad (verbos y conectores), siendo menos frecuente el abordaje de la estructura del discurso narrativo y, más aún, de la complejidad sintáctica del mismo.

Una de las líneas de investigación más aportadora en la comprensión del desarrollo narrativo infantil son los estudios de Labov y Waletzky (1967), quienes conceptualizan la narración como una secuencia de al menos dos cláusulas ordenadas temporalmente e indagan en la presencia y funcionalidad de esta en discursos de carácter personal, en los que los sujetos incluyen el relato de acontecimientos o sucesos personales con la finalidad de respaldar o ejemplificar lo que están compartiendo en conversaciones cotidianas. El interés estaba puesto en cómo a través de las narraciones los hablantes evalúan su experiencia. Para ello, los autores distinguen dos tipos de funciones de la narración: referencial y evaluativa. No obstante, para efectos del presente estudio, resulta más pertinente la distinción de los constituyentes del discurso narrativo. En su modelo, Labov (1972) propone: resumen, orientación, complicación, evaluación, resolución y coda. Estos constituyentes se relacionan con 
otras propuestas teóricas que abordan el modo en que se organiza el discurso narrativo (Adam, 1992).

Por su parte, Slobin y Bocaz (1988), en el contexto de una investigación translingüística, estudian el modo en que los niños aprenden a usar las marcas de temporalidad en el discurso narrativo producido a partir de un estímulo visual. La muestra consideraba sujetos de 3 a 12 años dentro de los cuales se consideró un grupo de niños chilenos. El foco de análisis estaba puesto en cómo los niños construyen la temporalidad a través del uso de las formas verbales.

En el ámbito hispano, Sebastián (1991) desarrolla un estudio en el contexto de una investigación translingüística mayor que comparó 4 lenguas e incluyó producciones de niños entre 3 y 6 años. La finalidad era determinar el desarrollo de los sistemas temporales y aspectuales en el discurso infantil. Los resultados le permiten afirmar a la autora que, a edad muy temprana ( 3 años), los niños hacen uso de todas las formas verbales del español. No obstante, este uso no implica necesariamente que éstas hayan sido adquiridas de forma definitiva "ni que las funciones atribuidas a esa forma por parte de los niños sean las mismas que las de los adultos" (Sebastián, 1991: 194). Este manejo precoz de las formas verbales no se manifiesta en la organización del discurso, que sigue siendo precario. Este hallazgo permite a la investigadora afirmar que "hasta los 5 años no podemos considerar que comienza a desarrollarse la capacidad narrativa" (Sebastián, 1991: 195).

Tolchinsky (1990) hace una revisión del concepto de narración desde aproximaciones psicológicas, literarias y lingüísticas. Este es un estudio de producciones escritas de 62 niños israelíes de 5 a 7 años (kínder a $3^{\circ}$ básico) que tiene por finalidad evidenciar la organización sintáctica de 1os textos y explorar la relación entre ésta y las diferentes funciones y contrastes narrativos. A nivel sintáctico, la autora concluye que:

el número de posiciones incluidas en la secuencia máxima crece con la edad del grupo; es decir, la construcción se torna más compleja. Los niños más pequeños usan construcciones verbales, o construcciones con elementos asociados o con elementos regidos. En cambio los mayores, nuestros sujetos de 7 años, llegan a incluir todas las posiciones sintagmáticas mencionadas. (Tolchinsky, 1990: 77) 
En el plano semántico, afirma que la construcción de los relatos en la infancia obedece más a una organización local, dada por los sucesos que se van presentando, que al tema general o macroestructura de los mismos y que esta organización emerge a medida que se va enunciando el discurso, lo que se expresa en una coincidencia entre el orden del relato y el de su enunciación. Y, a nivel discursivo, constata que, si bien los más pequeños organizan la narración como una serie de acontecimientos sin mayor estructura, logran construir un "enmarque temporal" y describir brevemente los sucesos.

En una investigación más reciente, Sánchez, Silva y Borzone (2009), estudian el empleo de tiempos verbales en narraciones orales y escritas de 163 niños de $1^{\circ}$ y $2^{\circ}$ básico, constatando que en ambas modalidades comunicativas los sujetos manifiestan un uso adecuado de los tiempos verbales, aun cuando los discursos sean más extensos en la oralidad. Los resultados, además, demuestran que la construcción del relato obedece más a la sumatoria de enunciados aislados que a relaciones temáticas más profundas. En la modalidad oral, aparecen más formas verbales y el uso del presente en formas coloquiales para abrir y cerrar los relatos.

\subsection{Estudios sobre desarrollo sintáctico}

Los estudios sobre desarrollo sintáctico han preferido la modalidad escrita (Hunt, 1970; Véliz, 1988, 1999), asociando la escolaridad al aprendizaje de lo escrito y situando el desarrollo oral en un segundo plano. Es así como los estudios del desarrollo sintáctico de la oralidad son más bien recientes, como se puede apreciar en los trabajos de Berman (2004), de Coloma, Peñaloza y Fernández (2007), de Katzenberger (2003) y de Nir-Sagiv \& Berman (2010).

La mayoría de los estudios de la complejidad sintáctica adoptan una línea de análisis vinculada a las propuestas de Hunt (1970), quien estableció índices de carácter cuantitativo mediante la conceptualización de la Unidad-T con el fin de estudiar la producción escrita de hablantes cuya lengua materna era el inglés. Esta medida establece el promedio de longitud de la unidad mínima terminal que consiste en una cláusula principal más las cláusulas que se adjuntan o se incrustan en ella. Por una parte, es mínima porque constituye la unidad más pequeña en que puede ser dividido un discurso sin dejar ningún fragmento como residuo. $\mathrm{Y}$, por otra, se llama terminal porque es gramaticalmente aceptable 
empezar cada una con una mayúscula y terminar con un punto o signo de interrogación (Benítez, Meneses y Ow, 2012).

Así, por ejemplo, Gutiérrez Clellen (1994), al investigar la complejidad sintáctica oral desde una perspectiva evolutiva, analiza producciones narrativas de 77 escolares de entre 5 y 8 años utilizando los índices de Hunt (1970). Los resultados de su estudio ponen de manifiesto que con la edad aumenta el largo de la unidades T, se incluye mayor cantidad de estructuras subordinadas y los sujetos producen discursos con mayor presencia de cláusulas de relativo y frases preposicionales. El autor señala que no obstante estos resultados, es importante considerar el tipo de tarea a la que son expuestos los sujetos, dado que la extensión de las unidades $\mathrm{T}$ demuestra ser sensible a la tarea de elicitación utilizada.

En el contexto chileno, Véliz (1988) estudió la complejidad sintáctica de producciones narrativas escritas de escolares chilenos de distintos niveles escolares entre $4^{\circ}$ básico y $\mathrm{IV}^{\circ}$ medio, haciendo una adaptación de los índices propuestos por Hunt (1970): unidad T, longitud de cláusula e índice de subordinación. Los resultados apuntan a un aumento de todas las unidades a medida que avanza el nivel de escolaridad de los sujetos. A mayor nivel escolar, los sujetos tienden a escribir unidades-T y cláusulas más largas y a incrustar, en una proporción mayor, cláusulas al interior de las unidades-T. Estos resultados le permitieron establecer un paralelismo entre los estudios de Hunt y los resultados de esta investigación. En 1991, un nuevo estudio confirma los hallazgos y los relaciona con distintas modalidades discursivas: argumentativa, descriptiva y narrativa, constándose que el modo narrativo tiene menor complejidad que el argumentativo, pero mayor que el descriptivo. Estos hallazgos apuntan a que en la narración las unidades-T son más breves y que la incrustación es menos usual que en la argumentación. Con estos resultados, Véliz (1988) afirma que el modo del discurso incide significativamente en el grado de complejidad sintáctica.

Por su parte, Coloma, Peñaloza y Reyes (2007) estudian el desarrollo sintáctico a partir del caso particular de las oraciones subordinadas en una muestra de 90 niños de $3^{\circ}$ y $5^{\circ}$ de Educación Básica en Chile. A partir de una tarea de recontado, concluyen que se produce un mayor uso de oraciones sustantivas a medida que aumenta la edad y que esta situación pareciera estar asociada a que los sujetos de 10 años tienden a construir los discursos referidos de manera indirecta, usando cláusulas sustantivas 
de subjunción "que". En cambio, los niños de 8 años lo hacen de manera directa, es decir, con oraciones yuxtapuestas. Los resultados, además, los llevan a sugerir que, en el análisis de las narraciones orales, debieran considerarse de modo especial las cláusulas que se vinculan con el acto de relatar (temporales, causales, consecutivas y de lugar).

Aun cuando los análisis mencionados constituyen un aporte valioso al estudio del desarrollo sintáctico oral, en las últimas dos décadas, las investigaciones sobre madurez sintáctica han complementado la aproximación formal con una funcional-discursiva.

Desde los estudios de Hunt (1965) hasta los primeros estudios realizados por Berman (1996) y Katzenberger (1999), la orientación de éstos ha cambiado y, por ende, ha transformado la perspectiva que se tenía de lo que se denomina madurez sintáctica. La visión actual es considerar esta madurez ya no sólo como una concatenación de cláusulas y palabras que se subordinan a medida que se produce el texto, sino más bien se considera como parte de una relación de significados al interior de unidades más amplias que una simple oración. (Benítez, Meneses y Ow, 2012: 70)

\subsection{Perspectiva funcional discursiva en el estudio de la complejidad sintáctica}

Desde una aproximación funcional discursiva, el análisis de la complejidad sintáctica va a estar dado por cuán apropiadamente se construye un texto a partir de unidades discursivas mayores a la oración que involucran criterios sintácticos, discursivos y temáticos que le dan cohesión más allá de las estructuras que se emplean. Más que constatar la presencia de determinadas formas lingüísticas -sean estas más o menos complejas- lo que importa es estudiar cómo se emplean estas formas para satisfacer determinadas funciones discursivas, bajo el supuesto de que una misma forma lingüística puede cumplir funciones diferentes (Berman, 2004, 2007; Katzenberger, 2003). Asimismo, este uso está en estrecho vínculo con las modalidades discursivas, pues no se construye sintácticamente del mismo modo un texto narrativo que uno explicativo, por ejemplo.

En este estudio, se ha operacionalizado la complejidad sintáctica adaptando el trabajo realizado por Berman y Nir-Sagiv (2009) y Nir y 
Berman (2010). Dicha propuesta establece como unidad de análisis la cláusula, la cual, a su vez, está inserta dentro de paquetes clausulares (Katzenberger, 2004) entendidos como unidades que incorporan criterios sintácticos, temáticos y discursivos. La complejidad se define así por el tipo de vínculo que establecen las cláusulas al interior de los paquetes clausulares. Siguiendo las distinciones de Berman y Nir-Sagiv (2009) y Nir y Berman (2010), se han determinado 6 tipos de relaciones interclausulares:

1. Isotaxis: cláusulas autónomas que no establecen relaciones sintácticas de subordinación o de dependencia con otras cláusulas.

2. Parataxis simétrica: cláusulas que establecen relaciones de equivalencia con otras mediante encadenamiento de información que se encuentra a un mismo nivel informativo.

3. Parataxis asimétrica: las cláusulas que establecen una relación de coordinación o de dependencia donde la segunda cláusula solo puede ser interpretada en relación con la principal, pero no está subordinada a ella.

4. Hipotaxis: cláusulas subordinadas a otra cláusula estableciendo una relación de dependencia.

5. Endotaxis: cláusulas anidadas dentro de otras cláusulas. Dentro de este grupo, se consideran las cláusulas relativas y adverbiales embebidas, cláusulas parentéticas.

6. Endotaxis reparadora: cláusulas que no contribuyen al desarrollo del contenido del discurso, sino que dan cuenta del proceso de enunciación y del sujeto que desarrolla la tarea.

La complejidad de estas relaciones es creciente, de modo que la aparición de una endotaxis en el discurso implica un desarrollo sintáctico superior si se lo compara con el uso de parataxis e hipotaxis. Además, hay relaciones que tienen mayor presencia dependiendo de la modalidad discursiva. En principio, pareciera que son más propias de las narraciones la parataxis simétrica y asimétrica, mientras que la hipotaxis y la endotaxis surgen con más frecuencia en discursos explicativos vinculados al conocimiento. 


\subsection{La secuencia narrativa}

La secuencia discursiva es una unidad de meso nivel que explica cómo se organizan los textos empíricos no constituidos por un solo tipo de estructura (Van Dijk, 1980). Corresponden a unidades relativamente autónomas que articulan macroproposiones diversas. De allí que un texto se conciba como una integración lineal de diferentes y diversas secuencias. En el caso de la secuencia narrativa, lo que la distingue de una simple sucesión de hechos es la presencia de una intriga o conflicto que modifica una situación inicial y que gatilla la acción de los personajes (Bronckart, 2004). Si bien existen varias propuestas que explican cómo se organiza una secuencia narrativa, en este estudio se ha utilizado la teoría de Adam $(1992,1999)$ que propone un esquema quinario:

\begin{tabular}{|lll|}
\hline Proposición Narrativa & Función Discursiva \\
\hline PN1 & Situación Inicial & $\begin{array}{l}\text { Se parte de una situación estable. Se } \\
\text { presentan los personajes principales, el } \\
\text { escenario y la época. }\end{array}$ \\
PN2 Conflicto & $\begin{array}{l}\text { Complicación que modifica el estado } \\
\text { precedente y desencadena el relato. }\end{array}$ \\
PN3 & Reacción & $\begin{array}{l}\text { Una reacción, evaluación mental o accional } \\
\text { de los que de una u otra forma han sido } \\
\text { afectados por la PN2. }\end{array}$ \\
PN4 & Desarrollo & $\begin{array}{l}\text { Una resolución o nuevo elemento } \\
\text { modificador, aparecido en la situación creada } \\
\text { a partir de la reacción a PN2. }\end{array}$ \\
PN5 & Situación Final & $\begin{array}{l}\text { Una situación final, donde se establece un } \\
\text { estado nuevo y diferente del primero. }\end{array}$ \\
\hline
\end{tabular}

\section{Tabla 1. Secuencia narrativa}

\section{Metodología}

El estudio forma parte de una investigación mayor sobre desarrollo sintáctico tardío en Chile (2010-2012), cuya finalidad es validar las tareas de elicitación de discursos orales narrativos y explicativos y el dispositivo de análisis construido a partir de la tipología de arquitectura interclausular propuesta por Ruth Berman y su equipo. El estudio mayor es de carácter correlacional-inferencial y su diseño es no experimental, 
transaccional, expo facto, retrospectivo simple. Se optó por este diseño en virtud de las limitaciones de trabajar con instituciones educativas que colaboran voluntariamente y de la dificultad derivada de la asignación aleatoria. Además, la investigación considera el establecimiento de relaciones de covariación entre variables. El uso de un diseño no experimental permite trabajar con los sujetos en un contexto natural y favorece la validez externa.

Se tomó una muestra de 259 estudiantes de 5 (NT2), $8\left(3^{\circ}\right), 11\left(6^{\circ}\right), 14$ $\left(\mathrm{I}^{\circ}\right)$ y $17\left(\mathrm{IV}^{\circ}\right)$ años de establecimientos educativos urbanos, de régimen mixto, con escolaridad completa y que estuvieran en la media en el SIMCE 2008 de Lenguaje. El análisis de la complejidad sintáctica en los discursos narrativos se realizó sobre la muestra total, mientras que el estudio del rol de los paquetes en relación con la secuencia narrativa fue desarrollado en una selección aleatoria de 3 casos de cada nivel escolar (15 casos en total).

Para medir el desarrollo sintáctico, se construyeron dos tareas para elicitar discurso oral. Por una parte, se presentó a los niños una explicación (disertación) sobre el porqué existen las escuelas. Por otra parte, una narración (cuento) sobre la visita de un extraterrestre a la escuela. Ambos estímulos fueron de carácter audiovisual, mezclando el texto verbal con una secuencia de imágenes especialmente diseñadas para este propósito. En este trabajo, se presentan los análisis y resultados de la aplicación de la tarea de narración.

Cada tarea se aplicó de manera individual. Los participantes observaban los videosy luego recontaban lo quehabían visto. Los discursos fueron grabados digitalmente y transcritos siguiendo las convenciones propuestas por Calsamiglia y Tusón (2002). La transcripción de cada producción oral fue segmentada en paquetes clausulares (Katzenberger, 2004) teniendo como referencia el texto fuente. Para la determinación de la complejidad sintáctica, los discursos narrativos fueron segmentados en paquetes clausulares; al interior de estos se identificaron y codificaron las relaciones interclausulares encontradas. A cada relación se asigna un puntaje que se relaciona con su nivel de complejidad, lo que permite establecer un índice por sujeto, por nivel educativo y por discurso, en este caso, el narrativo.

Para el estudio del rol de los paquetes clausulares en función de la secuencia narrativa, se seleccionaron tres casos de cada nivel educativo, 
de diversas instituciones educativas, género, extensión y complejidad sintáctica. Se segmentaron los textos en función de las proposiciones narrativas y se analizaron los roles que cumplía cada uno de los paquetes clausulares en estas, teniendo como referente el texto fuente al que fueron expuestos los participantes y las fases narrativas propuestas por Adam (1999). En algunos casos, se encontró una relación isomórfica entre ambas unidades (proposición narrativa y paquete clausular) y en otros se constataron dos situaciones: o bien que un paquete clausular podía dar origen a más de una proposición narrativa, o bien que una proposición incluía dos o más paquetes.

\section{Resultados}

La confiabilidad de consistencia interna del índice para dar cuenta de la complejidad sintáctica del discurso oral narrativo fue probada con el Alpha de Cronbach (Cronbach 2004), obteniéndose un resultado de 0,688. Según Ruiz Bolívar (1998), el resultado obtenido en esta prueba da cuenta de un nivel de confiabilidad alto.

\begin{tabular}{|l|c|c|c|c|}
\hline & \multicolumn{2}{|c|}{ Todos los ítems } & \multicolumn{2}{c|}{ Con ítems $\mathbf{r}>\mathbf{0 , 2}$} \\
\hline Texto & $\begin{array}{c}\text { Alpha } \\
\text { Cronbach }\end{array}$ & $\begin{array}{c}\text { Spearman - } \\
\text { Brown }\end{array}$ & $\begin{array}{c}\text { Alpha } \\
\text { Cronbach }\end{array}$ & $\begin{array}{c}\text { Spearman - } \\
\text { Brown }\end{array}$ \\
\hline Narrativo & 0,688 & 0,747 & 0,685 & 0,747 \\
\hline
\end{tabular}

Tabla 2. Coeficientes alpha de Cronbach y Spearman-Brown para el índice de complejidad sintáctica

Como se aprecia en el gráfico 1, los estudiantes construyen sus discursos narrativos con un creciente número de paquetes clausulares y con un uso cada vez más complejo de las claúsulas. En kínder, por ejemplo, las cláusulas que predominan son las paratácticas simétricas, situación que permanece en todos los niveles, pero que se combina de forma más equilibrada con otro tipo de construcciones, produciéndose un aumento progresivo de la parataxis asimétrica, de la hipotaxis y endotaxis. Un dato interesante, aunque de escasa presencia, es la incapacidad de algunos alumnos del nivel inicial (kínder, 5 años) de recontar la narración a la que fueron expuestos, lo que se expresa en la ausencia de isotaxis. 


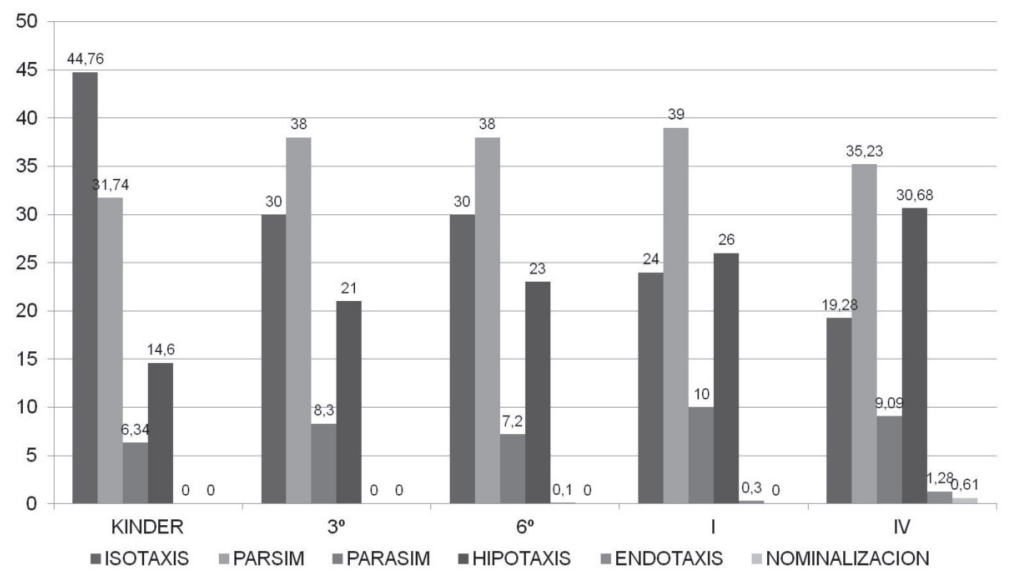

\section{Gráfico 1. Presencia de relaciones interclausulares por nivel escolar}

El uso de las cláusulas hipotácticas manifiesta un salto cuantitativo en $3^{\circ}$ básico, probablemente producido por el acceso a la cultura escrita y al conocimiento escolar. Esta situación varía escasamente en el siguiente nivel evaluado: $6^{\circ}$ básico, pero sí lo hace en el primer nivel de la Enseñanza Media, en el que se consolida el uso de la endotaxis. Asimismo, en el último curso, IV medio, aparece un fenómeno nuevo: la nominalización, que si bien involucra una dimensión sintáctica, también supone una transmorfologización y una condensación de información con el consiguiente aumento de la densidad léxica (Cinto, 2009).

\subsection{Rol de los paquetes clausulares en la secuencia narrativa}

A continuación, se presentan las tablas con los resultados de los análisis para determinar el rol de los paquetes clausulares dentro de la secuencia narrativa:

\begin{tabular}{|l|c|c|c|c|c|}
\hline \multicolumn{5}{|c|}{ PAQUETE CLAUSULAR 1 } \\
\hline & PN1 & PN2 & PN3 & PN4 & PN5 \\
\hline Kinder & & $X$ & & & \\
\hline $3^{\circ}$ Básico & $X$ & $X$ & & \\
\hline $6^{\circ}$ Básico & $X$ & $X$ & & \\
\hline I $^{\circ}$ Medio & $X$ & $X$ & & \\
\hline IV $^{\circ}$ Medio & $X$ & & & \\
\hline
\end{tabular}

Tabla 3. Rol del paquete clausular 1 en la secuencia narrativa 
En la tabla 3, se puede observar que el paquete clausular 1 en el kínder coincide con la proposición narrativa 2, correspondiente a la introducción del conflicto y complicación. Por su parte, tanto $3^{\circ}$ básico, $6^{\circ}$ básico y $\mathrm{I}^{\circ}$ medio, presentan una correspondencia entre el paquete clausular 1 y las proposiciones narrativas 1 (correspondiente a la presentación de personajes principales, escenario y época) y proposición narrativa 2 (introducción al conflicto y complicación). Finalmente, se puede constatar que sólo el IV medio tiene una correspondencia en las muestras analizadas del paquete clausular 1 con la proposición narrativa 1.

\begin{tabular}{|c|c|c|c|c|c|}
\hline \multicolumn{6}{|c|}{ PAQUETE CLAUSULAR 2} \\
\hline & PN1 & PN2 & PN3 & PN4 & PN5 \\
\hline Kinder & \multicolumn{5}{|c|}{$\begin{array}{l}\text { No tiene uso específico. Más elementos de PN2 y PN4. } \\
\text { En un caso, coincidió con PN5. }\end{array}$} \\
\hline $3^{\circ}$ Básico & & $X$ & & $X$ & \\
\hline $6^{\circ}$ Básico & & $X$ & & $X$ & \\
\hline $\mathrm{I}^{\circ}$ Medio & \multicolumn{5}{|c|}{$\begin{array}{l}\text { No tiene un uso específico. Presenta mayores } \\
\text { elementos de PN4 }\end{array}$} \\
\hline $\mathrm{IV}^{\circ}$ Medio & & $X$ & $X$ & & \\
\hline
\end{tabular}

Tabla 4. Rol del paquete clausular 2 en la secuencia narrativa.

\begin{tabular}{|c|c|c|c|c|c|}
\hline \multicolumn{6}{|c|}{ PAQUETE CLAUSULAR 3-4-5... } \\
\hline & PN1 & PN2 & PN3 & PN4 & PN5 \\
\hline Kinder & \multirow{5}{*}{\multicolumn{5}{|c|}{$\begin{array}{l}\text { En todos los niveles se constató que no tienen un uso } \\
\text { específico, gran variabilidad, existen más elementos } \\
\text { de PN3 y PN4. En algunos casos, especialmente, en los } \\
\text { cursos menores de escolarización, corresponde al PN5. }\end{array}$}} \\
\hline $3^{\circ}$ Básico & & & & & \\
\hline $6^{\circ}$ Básico & & & & & \\
\hline $\mathrm{I}^{\circ}$ Medio & & & & & \\
\hline$V^{\circ}$ Medio & & & & & \\
\hline
\end{tabular}

Tabla 5. Rol del paquete clausular 3, 4, 5 y otros en la secuencia narrativa. 


\begin{tabular}{|l|c|c|c|c|c|}
\hline \multicolumn{9}{|c}{ ÚLTIMO PAQUETE CLAUSULAR } \\
\hline & PN1 & PN2 & PN3 & PN4 & PN5 \\
\hline Kinder & & & & & X \\
\hline $3^{\circ}$ Básico & & & & & X \\
\hline $6^{\circ}$ Básico & & & & & X \\
\hline$I^{\circ}$ Medio & & & & & X \\
\hline IV $^{\circ}$ Medio & & & & & X \\
\hline
\end{tabular}

Tabla 6: Rol del paquete clausular final en la secuencia narrativa.

\subsection{Función de las relaciones interclausulares en los PC inicial y final}

Para determinar, de una forma preliminar, la función del tipo de relación sintáctica que utilizan los niños en el discurso narrativo, se seleccionó el PC inicial y final, pues constituyen los paquetes que mantuvieron en todos los niveles una constante de uso. En base a lo anterior, se llegó a determinar que, en el caso de los PC iniciales, predominan las oraciones subordinadas relativas y, en cambio, en los PC finales, predominan las construcciones paratácticas asimétricas en los niveles superiores de escolarización. En los niveles iniciales, predomina el uso de la ISOuni.

\begin{tabular}{l|l|l|}
\hline \multicolumn{1}{|c|}{ PC Inicial } & \multicolumn{3}{|c|}{ PC Final } \\
$\begin{array}{l}\text { Predominan las subordinadas } \\
\text { relativas. }\end{array}$ & $\begin{array}{l}\text { Predominan las parataxis } \\
\text { simétricas. } \\
\text { En los niveles iniciales también la } \\
\text { ISOuni. }\end{array}$ \\
\hline $\begin{array}{l}\text { Función: Presentación de los } \\
\text { personajes y espacios. }\end{array}$ & $\begin{array}{l}\text { Función: Presentación de la } \\
\text { situación final }\end{array}$ \\
$\begin{array}{l}\text { Ejemplo: } \\
\begin{array}{l}\text { (PC1) Em:: habí...a ... [Falso } \\
\text { comienzo] un día vino [ISOprin]:: } \\
\text { un extraterrestre que, que les } \\
\text { enseñó [HIPOadj] a los niños } \\
\text { harto }\end{array}\end{array}$ & $\begin{array}{l}\text { Ejemplo: } \\
\text { [ISOprin] } \\
\text { ynoledijeronchao[SIMcoordSIEl] } \\
\text { y se fue [SIMcoordSIEl] }\end{array}$ \\
\hline
\end{tabular}

Tabla 7. Función de las relaciones interclausulares en los PC inicial y final. 


\subsection{Relación anisomórfica entre los PC y la secuencia narrativa}

A continuación, se presentan las relaciones anisomórficas entre los paquetes clausulares y la secuencia narrativa manifestada en la muestra analizada:

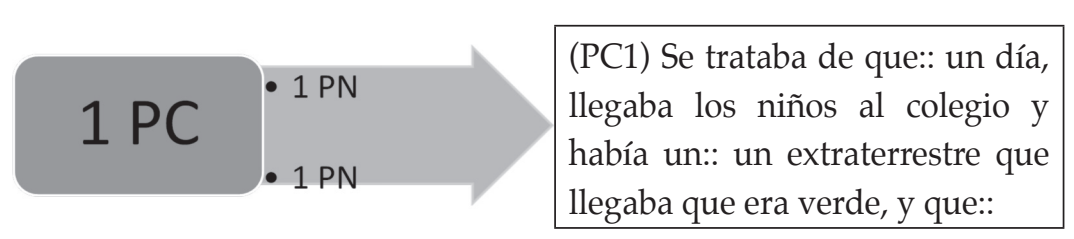

La primera relación anisomórfica muestra cómo dentro de un mismo paquete clausular se manifiestan dos proposiciones narrativas. En este ejemplo, se da cuenta del PC1, el cual coincide con la PN1: "Se trataba [ISOprin] de que:: un día, llegaba [ASIMcomp] los niños al colegio" y PN2: "había [SIMcoordSDEx] un:: un extraterrestre que llegaba [HIPOadj] que era verde [HIPOadj], y que::"

\begin{tabular}{|l|l|}
\hline (PC8) eh:: entonces empieza:: \\
a/ em:: [Falso comienzo] \\
debido debilitamiento no puede \\
comunicarse, no puede entender \\
lo que le dicen los otros y él no \\
puede comunicar lo que siente, \\
entonces se siente muy mal y por \\
eso decide marcharse a su planeta \\
una mañana igual como él llegó \\
y:: muy triste por no poder decir \\
por no poder despedirse de sus \\
amigos/ \\
(PC9) y desde y desde cuando él \\
va volando por lo visto le gritan \\
lo::s/ los humanos chao en el \\
idioma nativo de ellos
\end{tabular}


La segunda relación anisomórfica da cuenta cómo una proposición narrativa se distribuye en dos o más paquetes clausulares. En este ejemplo, se muestra que la PN5 está distribuida en dos paquetes clausulares: el PC8 y el PC9.

\section{Conclusiones}

Las conclusiones de este trabajo pueden esbozarse considerando diversos aspectos de la experimentación. De esta manera, podemos tener en cuenta cuatro focos centrales: la eficacia de la tarea de elicitación; el valor de la secuencia de Adam para entender el desarrollo discursivo de los alumnos; la noción de paquete clausular y su valor intermedio entre el nivel sintáctico y el tipo de texto. Y, finalmente, la complejidad sintáctica y cómo esta parece condicionada por distintos aspectos de lo discursivo.

Con respecto a la tarea de elicitación, se pueden hacer varias afirmaciones. Por un lado, se puede señalar que la elaboración de una tarea de recontado con estímulo visual y auditivo permite elicitar discurso independiente de cuál sea la edad de los sujetos. De esta manera, se pudo homogeneizar una sola tarea a través de los distintos grupos de edad y esto permitió que la comparación entre discursos fuera posible. Por otro, es necesario señalar que siguiendo lo propuesto por Berman (2004), la tarea exigida guardaba cierta similitud con las tareas habituales que llevan los niños en el colegio, ya que es en este ámbito en donde es más evidente el desarrollo del lenguaje oral tardío. En otras palabras, la generación de una tarea experimental, de carácter monogestionado y temática ficticia resulta productiva para la elicitación del discurso narrativo oral en niños y adolescentes.

Considerar para este estudio la secuencia narrativa propuesta por Adam $(1992,1999)$ ha permitido revisar la segmentación realizada en función de los paquetes clausulares. Esta revisión podría influir en los índices de complejidad sintáctica, pues lo que Adam llama una macroproposición puede equivaler o no a un PC. Se pudo establecer que los sujetos, independiente de la edad, manifiestan un manejo de las fases fundamentales de la secuencia narrativa: Complicación y Situación final. A medida que avanzan en edad, incorporan las otras fases: Situación inicial, Nuevo elemento modificador y Reacción a la complicación. 
El rol del primer PC varía según la edad, los estudiantes de Kínder (probablemente menos expuestos por edad y contexto a narraciones literarias) usan este PC para introducir el conflicto, mientras que, a partir de $3^{\circ}$ básico en adelante, se constata que es usado como presentación de la situación inicial, pero generalmente coincidiendo también con otra proposición narrativa. Sólo los estudiantes de $\mathrm{IV}^{\circ}$ Medio evidenciaron la correspondencia del uso del primer paquete clausular con la PN1. De las tres fases de la secuencia narrativa propuesta por Adam (1992, 1999), la que presenta menor manejo es la PN3 (reacción a la complicación). Esto puede deberse no solo a su complejidad propia dentro de la narración (dada por su posición) y su menor valor para el funcionamiento de la narración, sino también a la brevedad que fue presentada en el texto original de la tarea. Generalmente, aparece incluida con otra proposición, pero no sola como paquete clausular. Se constata una mayor extensión en el desarrollo de los paquetes clausulares de cierre, incluyéndose en algunos casos más de un PC en la PN5: Situación final. Una probable hipótesis es la mayor posibilidad de retener en memoria los detalles de la narración original.

Respecto a la complejidad sintáctica, los resultados iniciales de este estudio confirman hallazgos anteriores. Por una parte, se corrobora que ésta avanza con la edad y los niveles de escolaridad, desde relaciones clausulares paratácticas simétricas, a asimétricas, hipotácticas y endotácticas. Sin embargo, se ha detectado que algunas relaciones hipotácticas comienzan a ser utilizadas desde temprana edad, pues más del 50\% de los estudiantes de kínder la usan. No así las relaciones paratácticas asimétricas que están más descendidas en este nivel en comparación a las hipotácticas. Esto permitiría plantear en primera instancia que la subordinación sustantiva es más tardía en aparición y, por ende, en complejidad que ciertas subordinaciones adverbiales. Finalmente, la endotaxis aparece esporádicamente en muestras de niveles intermedios, no pudiéndose constatar una tendencia.

Por otra parte, resulta determinante para este estudio establecer la articulación entre complejidad sintáctica y género discursivo. En este caso, se pudo determinar que el uso de subordinadas de relativo aparece desde los niveles iniciales de escolarización, principalmente, en el PC inicial con la función de presentar la situación narrativa. En este sentido, se puede corroborar que el tipo de género discursivo va a favorecer el uso constante de ciertas estructuras sintácticas. Asimismo, se ha 
podido observar de forma preliminar que la segmentación en paquetes clausulares permite distinguir qué tipos de relaciones interclausulares se dan y su función dentro del paquete clausular en relación al género discursivo.

\section{Bibliografía}

Adam, J. M. (1992). Les textes: types et prototypes. Récit, description, argumentation, explication et dialogue. París: Nathan.

Adam, J. M. (1999). Linguistique textuelle. Des genres de discours au textes. París: Ed. Nathan.

Benítez, R.; Meneses, A. y Ow, M. (2012). “Complejidad sintáctica: ¿modalidad comunicativa o tipo textual? Estudio de casos de producciones textuales de estudiantes de $5^{\circ}$ básico". Onomázein 25, 65-93.

Berman, R. (2004). "Between emergence and mastery: The long developmental route of language acquisition". En R. Berman (Ed.), Language Development across Childhood and Adolescence, AmsterdamPhiladelphia: John Benjamins, 9-34.

Bocaz, A. (1996). "El paisaje de la conciencia en la producción de narraciones infantiles". Lenguas Modernas 23, 49-70.

Bruner, J. (2004). "Life as narrative”. Social Research 71 (3), 691-710.

Bronckart, J.P. (2004). Actividad verbal, textos y discursos. Por un interaccionismo socio-discursivo. Madrid: Fundación Infancia y Aprendizaje.

Calsamiglia, H. \& Tusón, A. (2002). "La textura discursiva”. Las cosas del decir. Manual de análisis del discurso. H. Calsamiglia \& A. Tusón (Eds.). Barcelona: Ariel, 217-250.

Cinto, M. T. (2009). “La nominalización. Obstáculo para la comprensión lectora". Educación, Lenguaje y Sociedad VI, 6, 37-59.

Coloma, C.; Peñaloza, C. y Fernández, R. (2007). "Producción de oraciones complejas en niños de 8 y 10 años". Revista de Lingüística Teórica y Aplicada 45, 33-44. 
Gutiérrez-Clellen, V. \& Hofstetter, R. (1994). "Syntactic complexity in spanish narratives". Journal of Speech and Hearing Research 37, 645-654.

Hunt, K. (1965). Grammatical structures written at three grade levels. Urbana, IL: National Council of Teachers of English. . (1970). "Syntactic maturity in school children and adults". Monographs of the Society of Research in Child Development 134 (35), 1, 33-45.

Jiménez, T. (2006). "La narración infantil. Un estudio en niños de educación básica". Revista de investigación 60, 157174.

Katzenberger, I. (2004). "The development of clause packaging in spoken and written texts". Journal of Pragmatics 36 (10), 1921-1948.

Labov, W. (1972). The transformation of experience in narrative syntax. Language in the inner city. Studies in the Black English Vernacular. Filadelfia: University of Pennsylvania Press.

\& Waletzky, J. (1967). "Narrative analysis". Essays on the Verbal and Visual Arts. J. Helm (Ed.). Seattle: U. of Washington Press, 12-44.

Nelson, K. (1996). Language in cognitive development. Cambridge: Cambridge University Press.

Ninio, A. \& Show, C. (1996). Pragmatic Development. Boulder: Westview Press.

Nir, B. \& Berman, R. (2010). “Complex syntax as a window on contrastive rhetoric". Journal of Pragmatics 42 (3), 744-765.

Pavez, M.; Coloma, C. \& Maggiolo, M. (2009). “El desarrollo narrativo en niños. Una propuesta práctica para la evaluación y la intervención en niños con trastornos del lenguaje". Onomázein 19, 155-157.

Ruiz Bolívar, C. (1998). Instrumentos de Investigación Educativa. CIDEG. Barquisimeto. Lara, Venezuela. 
Sánchez, V., Silva, M. L. \& Borzone, A. M. (2009). “El empleo de los tiempos verbales en la re-narración. Un estudio de las producciones orales y escritas de niños pequeños". Boletín de Lingüística XX, 95-117.

Sebastián, E. (1991). “El desarrollo de referencia temporal en español: Un paseo por la morfología verbal". Anales de Psicología 7 (2), 181-196.

\& Slobin, D. (1994). "Development of linguistic forms: Spanish". Relating Events in Narrative: A Crosslinguistic Developmental Study. R. Berman \& D. Slobin (Eds.). Hillsdale NJ: Lawrence Erlbaum, 239-284.

Slobin, D. \& Bocaz, A. (1988). "Learning to talk about the movement time and space: The development of narrative abilities in Spanish and English". Lenguas Modernas 15, 5-24.

Tolchinsky, L. (1990). “La reproducción de relatos en niños entre cinco y siete años: Organización sintáctica y funciones narrativas". Anuario de Psicología, Universidad de Barcelona 47, 67-87.

Uccelli, P. \& Paez, M.M. (2007). “Narrative and vocabulary development of bilingual children from kindergarten to first grade: Developmental changes and associations among English and Spanish skills". Language, Speech, and Hearing Services in Schools 38, 225-236.

Van Dijk, T. (1980). La ciencia del texto. Barcelona: Paidós.

Véliz, M. (1988). "Evaluación de la madurez sintáctica en el discurso". Revista de Lingüística Teórica y Aplicada 26, 105-141. (1999). "Complejidad sintáctica y modos del discurso". Estudios Filológicos 34, 181-192. 